\title{
A rare cause of ischemic proctosigmoiditis: idio- pathic myointimal hyperplasia of mesenteric veins
}

Ischemic proctosigmoiditis is an uncommon entity in colon ischemia because it is usually associated with an extensive arterial network. Here we report a case of idiopathic myointimal hyperplasia of the mesenteric veins (IMHMV), a very rare cause of mesenteric ischemia, which typically contributes to proctosigmoiditis.

A 60-year-old man presented with a 1month history of lower abdominal pain, bloody diarrhea, and weight loss of $10 \mathrm{~kg}$. A first colonoscopy in another hospital reported ulcerative colitis. The patient was treated with mesalazine initially, but with little clinical improvement.

Repeated colonoscopy 2 weeks later showed multiple ulcerations in the sigmoid colon and rectum up to $30 \mathrm{~cm}$ from the anal verge. Pathological analysis after endoscopic biopsy reported inflammatory cell infiltration of rectal and colonic mucosa. The patient received steroid therapy, balsalazide, and antibiotics, but his symptoms persisted.

Follow-up colonoscopy 10 days later continued to reveal multiple serpiginous circular ulcers between 30 and $10 \mathrm{~cm}$ from the anal verge ( Fig.1). Pathological analysis after endoscopic biopsy showed the presence of thick-walled, mediumsized blood vessels at the base of the ulcer, with mural hyalinization and focal thrombosis. Angiography showed no demonstrable opacification of the inferior mesenteric vein (IMV) ( Fig.2). Finally, a Hartmann's procedure of the affected part of the rectosigmoid colon was carried out. Grossly, the mucosal surface of the resected specimen showed diffuse ulceration with fibrinopurulent exudates (๑ Fig.3). Histologically, myointimal hyperplasia of the intramural and extramural mesenteric veins was noticed, with nearly total occlusion of the lumens and without an associated inflammatory infiltrate ( $\bullet$ Fig. 4 ).

The postoperative course was uneventful; the patient was free from abdominal cramps and bloody diarrhea. Follow-up colonoscopy 4 months later revealed normal colonic mucosa.

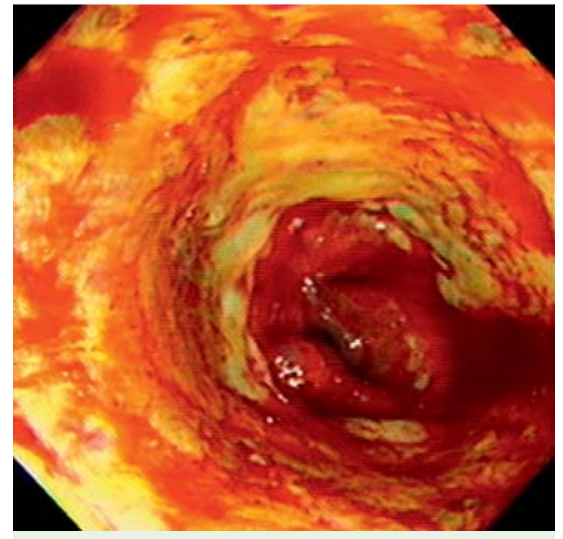

Fig. 1 Colonoscopy demonstrated multiple serpiginous circular ulcerations between 30 and $10 \mathrm{~cm}$ from the anal verge. The mucosa is edematous and friable, and is covered by copious amounts of mucoid discharge, seen as white, creamy material.

Non-thrombotic occlusion of the mesenteric veins is a rare cause of mesenteric ischemia, and IMHMV is the rarest entity among these causes. Patients with IMHMV usually present with a subacute course of weight loss, lower abdominal pain, diarrhea/constipation, and bloody stools. Clinically, these patients are often diagnosed with idiopathic inflammatory bowel disease (IBD), evidenced by the features under colonoscopy, and treated for IBD without an effective response. Mucosal biopsies, interestingly, show thick-

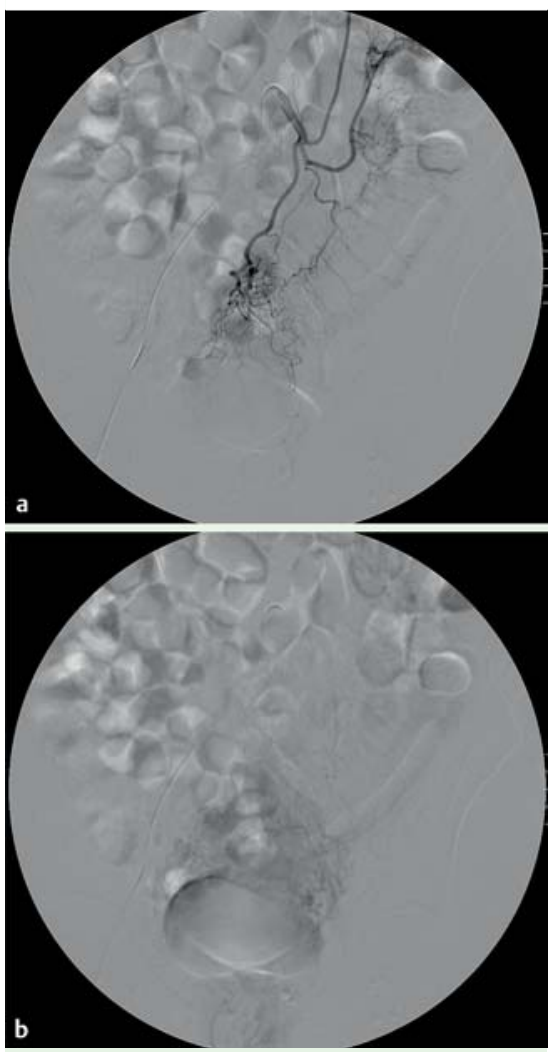

Fig.2 Angiography of the inferior mesenteric artery (IMA). a The opacification shows the IMA to have a smooth outline and good patency. b No demonstrable inferior mesenteric vein (IMV) is observed during venous-phase angiography, which indicates venous occlusion; however, no definite arteriovenous fistula was found in this study.

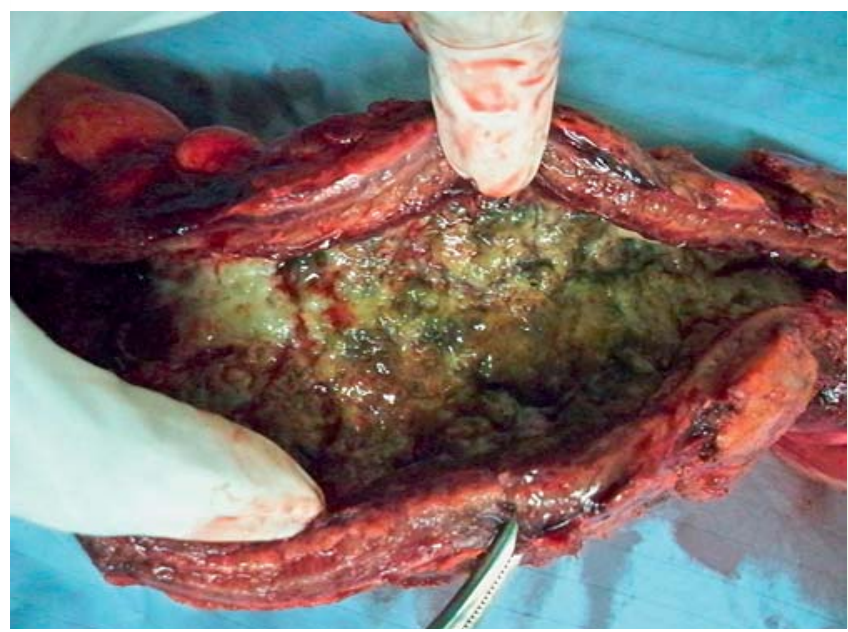

Fig. 3 The resected colon presented with thickened walls. The mucosal surface shows conspicuous segmental diffuse ulcerations with fibrinopurulent exudates. 

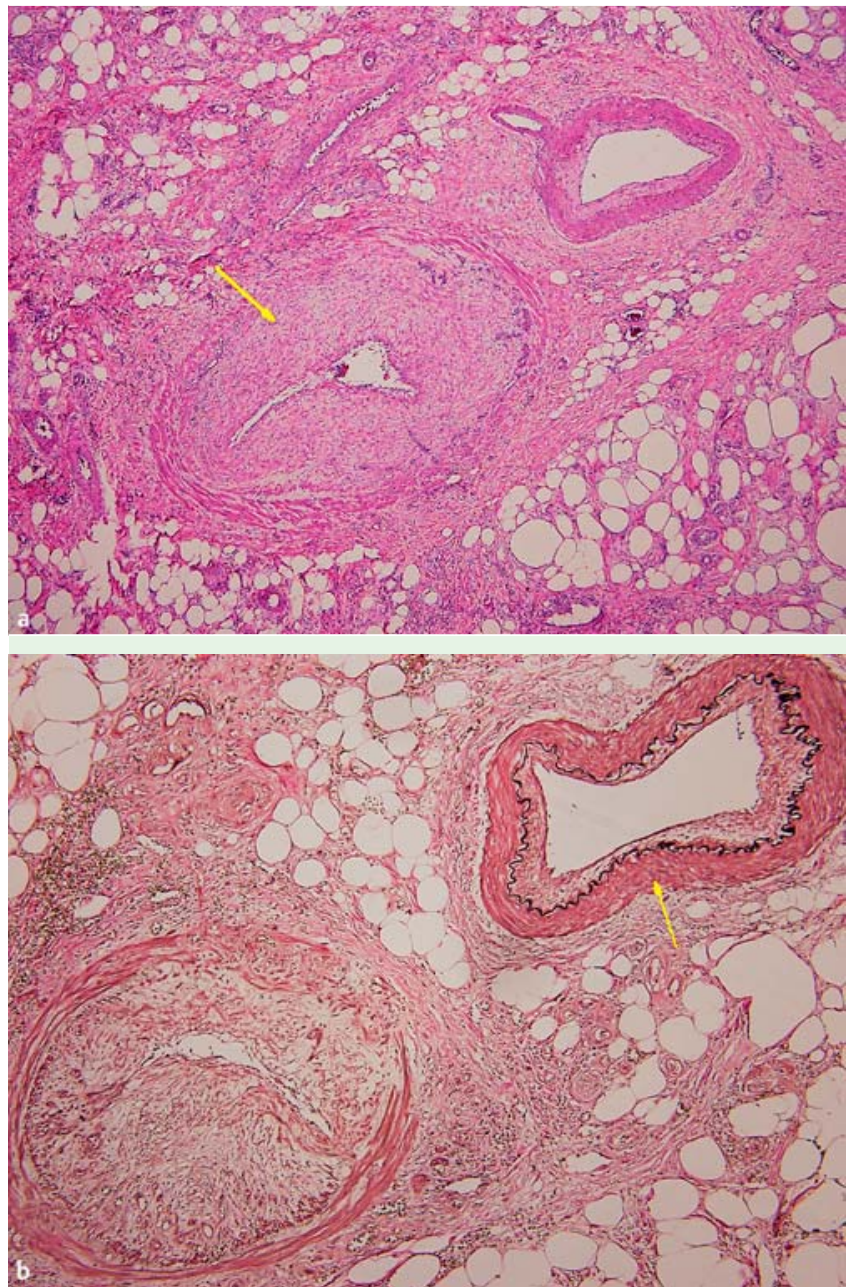

Fig. 4 Histopathological findings for the rectosigmoid resection specimen. a The mesenteric vein (arrow) shows near-total occlusion, marked myointimal hyperplasia, and even focal recanalization, but without an associated inflammatory infiltrate. The accompanying artery is spared (Hematoxylin and eosin stain $\times 100$ ). b Elastica van Gieson stain $(\times 200)$; this special elastin stain distinguishes the artery (arrow) from the vein, and is particularly helpful in the diagnosis of idiopathic myointimal hyperplasia of the mesenteric veins (IMHMV). walled vessels, focal ulcerations, or fibrin thrombi in the mucosa and submucosa, which do not suggest the diagnosis of IBD [1]. The mainstay of therapy is resection of the involved segment of colon, after which the prognosis is usually smooth. Extensive myointimal hyperplasia of the mesenteric veins in the submucosa, the adventitia, and the mesocolon - which reduces the lumens of the involved vessels, even to nearly total occlusion - is the pathognomonic feature [1]. To avoid misreading veins as arteries, a special elastin stain (Elastica Van Gieson) may be required [2].

The etiology of IMHMV remains unclear. Secondary arterialization of the mesenteric veins due to increased intravascular pressure may be the cause of myointimal hyperplasia, because of the closely similar histological findings in patients with failed saphenous vein coronary grafts and dialysis patients with stenosis of their arteriovenous fistulas $[1,3]$.

Although the postoperative course is uneventful in most cases, perforation of the resected colon has occurred in several cases $[2,4,5]$.

The average duration from onset of symptoms to surgery is about 6 months. This indicates the difficulty of diagnosing IMHMV.

Endoscopy_UCTN_Code_CCL_1AD_2AF

Competing interests: None

\section{C.-K. Chiang ${ }^{1}$, C.-L. Lee Le, $^{1,3}$, C.-S. Huang ${ }^{4}$, S.-H. Huang ${ }^{5}$, C.-H. Wu ${ }^{1}$}

${ }^{1}$ Division of Gastroenterology, Cathay General Hospital, Taipei, Taiwan

2 Division of Gastroenterology, Cathay

General Hospital, Hsinchu, Taiwan

${ }^{3}$ School of Medicine, Taipei Medical University, Taipei, Taiwan

${ }^{4}$ Department of Surgery, Cathay General Hospital, Taipei, Taiwan

${ }^{5}$ Department of Pathology, Cathay

General Hospital, Taipei, Taiwan

\section{References}

1 Abu-Alfa AK, Ayer U, West AB. Mucosal biopsy findings and venous abnormalities in idiopathic myointimal hyperplasia of the mesenteric veins. Am J Surg Pathol 1996; 20: $1271-1278$

2 Kao PC, Vecchio JA, Hyman NH et al. Idiopathic myointimal hyperplasia of mesenteric veins: a rare mimic of idiopathic inflammatory bowel disease. J Clin Gastroenterol 2005; 39: 704-708

3 Sherman J, Kao PC, Brian West A et al. Focal myointimal hyperplasia of mesenteric veins is associated with previous trauma in surgical specimens. Pathol Res Pract 2006; 202: 517-522

4 Genta RM, Haggitt RC. Idiopathic myointimal hyperplasia of mesenteric veins. Gastroenterology 1991; 101: 533-539

5 Savoie LM, Abrams AV. Refractory proctosigmoiditis caused by myointimal hyperplasia of mesenteric veins: report of a case. Dis Colon Rectum 1999; 42: 1093 -1096

\section{Bibliography}

DOI http://dx.doi.org/

10.1055/s-0031-1291529

Endoscopy 2012; 44: E54-E55

(c) Georg Thieme Verlag KG

Stuttgart · New York

ISSN 0013-726X

\section{Corresponding author}

\section{C.-L. Lee, MD}

Division of Gastroenterology

Department of Internal Medicine

Cathay General Hospital

Hsinchu

Taiwan

Fax: +886-3-5263199

cghleecl@hotmail.com 